\title{
Universal tunneling time for all fields
}

\author{
G. Nimtz ${ }^{1,2}$ and A. A. Stahlhofen ${ }^{1}$ \\ ${ }^{1}$ Institut für Integrierte Naturwissenschaften, Universität Koblenz \\ ${ }^{2}$ II. Physikalisches Institut, Universität zu Köln
}

October 25, 2018

\begin{abstract}
Tunneling is probably the most important physical process. The observation that particles surmount a high mountain in spite of the fact that they don't have the necessary energy can not be explained by classical physics. However, this so called tunneling became allowed by the theory of quantum mechanics. Experimental tunneling studies with different photonic barriers from microwave frequencies up to ultraviolet frequencies pointed toward a universal tunneling time [1, 2]. The observed results and calculations have shown that the tunneling time of opaque photonic barriers (for instance optical mirrors) equals approximately the reciprocal frequency of the electromagnetic wave in question. The tunneling process is described by virtual photons [3]. Virtual particles like photons or electrons are not observable. However, from the theoretical point of view, they represent necessary intermediate states between observable real states. In the case of tunneling there is a virtual particle between the incident and the transmitted particle. Tunneling modes have a purely imaginary wave number. They represent solutions of the Schrödinger equation and of the classical Helmholtz equation. The most prominent example of the occurrence of tunneling modes in optics is frustrated total internal reflection (FTIR) at double prisms. In 1949 Sommerfeld [4 pointed out that this pseudo classical optical phenomenon represents the analogy of quantum mechanical tunneling. Recent experimental and theoretical data confirmed the conjecture that the tunneling process is characterized by a universal tunneling time independent of the kind of field. Tunneling proceeds at a time of the order of magnitude of the reciprocal frequency of the wave.
\end{abstract}

Optical evanescent modes and solutions of quantum mechanical tunneling have a purely imaginary wave number. This means that they do not experience a phase shift in traversing space. The delay time $\tau$ of a propagating wave packet is given by the derivative

$$
\tau=-d \phi / d \omega
$$


where $\phi$ is the phase shift of the wave and $\omega$ is the angular frequency; $\phi$ is given by the real part of the wave number $k$ times the distance $x$. In the case of evanescent modes and tunneling solutions the real part of $k$ is zero. (The imaginary part is often called $\kappa$.) Thus propagation of evanescent modes appears to take place in zero time. In the case of particle tunneling Eq.1 is replaced by the corresponding derivative of the S-matrix with respect to energy.

Despite the fact that the semiconductor tunnel diode has been used since 1962 the particle barrier penetration time has not been determined up to now due to parasitic time consuming electronic interaction effects in a semiconductor. Around 1990 the mathematical analogy between the Schrödinger and the Helmholtz equations inspired microwave and optical tunneling experiments to obtain empirical data on the tunneling time. The experiments revealed superluminal (faster than light) signal and energy velocities [5, 6, 7, 8].

Several quantum mechanical (QM) and quantum electrodynamical (QED) calculations predicted that evanescent modes and tunneling of particles appear to propagate in zero time [3, 9, 11, 12, 13, 14]. However, there arises a scattering time at the barrier entrance in agreement with the Hartman effect. The latter states that the tunneling time does not depend on barrier length [9]. The effect was first confirmed by microwave experiments [10].

Superluminal tunneling experiments were carried out first with microwaves in undersized wave guides by Enders and Nimtz and later reproduced at microwaves and optical frequencies in photonic band gap material, i.e. optical mirrors [8, 15].

The tunneling time is of the order of magnitude of the inverse frequency of the tunneling wave. This property was observed for electromagnetic tunneling [1, 2, 8]. Recent experimental and theoretical results point to a universal tunneling time for all kinds of fields.

Zero time tunneling and the virtual nature of evanescent modes as well as the barrier interaction time become most obvious in FTIR with double prisms as shown in Refs. [3, 16, 17].

A modern technical device based on FTIR is illustrated in Fig 1. The two fibers (1) and (2) are wave guides of today's digital infrared communication systems [7]. The fibers represent light valleys. If they are brought to a narrow distance of a about a wavelength, more and more intensity of the infrared signals are tunneling from fiber (1) to fiber (2). Engineers call such a device a coupler, the inter-fiber distance determines the coupling ratio between the two guides by the tunneling process. Here, the barrier is given by an air gap.

In FTIR there is a shift (coined Goos-Hänchen shift) between the incident and the reflected beam as already conjectured by Newton 300 years ago.

The length of this shift amounts to about one wavelength [16]. This shift along the boundary of the first prism corresponds to the universal tunneling time of one oscillation time [1, 2]. The shift represents the interaction time at the boundary in the case that the beam has no normal incidence to the boundary.

The relationship for the tunneling intensity $I_{t}$ in the case of FTIR is 


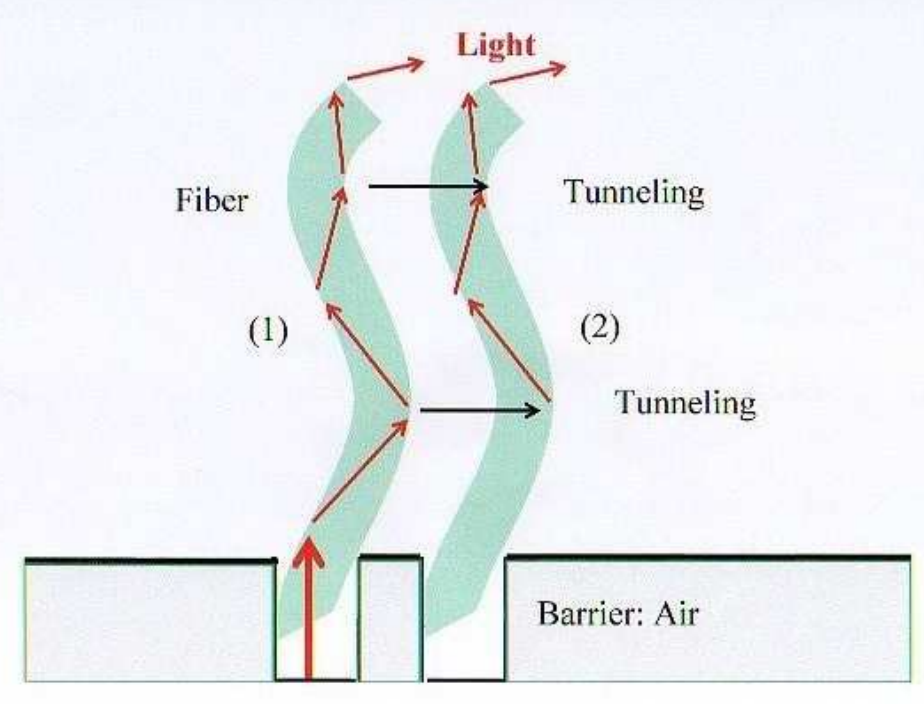

Figure 1: Sketch of two fibers (1) and (2). The guided infrared digital signal beam is totally reflected, however, tunneling takes placesince the two fibers are approaching each other. A fiber represents a light valley between the air barrier environment.

$$
\begin{aligned}
I_{t}(x) & =I_{0} e^{(i \omega t-2 \kappa x)} \\
\kappa & \left.=\left[\frac{\omega^{2}}{c^{2}}\left(\left(\frac{n_{1}}{n_{2}}\right)^{2}-1\right) \sin ^{2} \theta\right)\right]^{1 / 2},
\end{aligned}
$$

where $I_{0}$ is the the signal intensity at the barriers front, $\kappa$ is the imaginary wave number, $c, n_{1}$ and $n_{2}$ are velocity of light, the refractive indices of the air and of the fibers, respectively, and $\theta$ represents the angle of incidence above the critical angle of total reflection [16].

It is interesting that evanescent modes and tunneling particles are non observables. They can neither be detected in an undersized waveguide nor in a potential barrier for instance [3, 8]. This behavior is in consequence of the uncertainty relation [3]. The universal tunneling time seems to be valid also for sound waves as measured, for instance, by Yang et al. at a frequency of $1 \mathrm{MHz}$ and by Robertson et al. at $1 \mathrm{kHz}$ in a sound tunneling experimental set-up [18, 19]. Presumably, the virtual behavior of photons applies for all wave solutions having purely imaginary wave numbers independent of the kind of field. For instance electronic tunneling time values measured and discussed in field emission microscopy and in III-V compound semiconductors resulting in a time also fitting in the universal tunneling time scheme [22]. Table 1 shows tunneling data measured with different barriers and fields, where $\tau, \nu$ and $T$ are the tunneling time, the carrier frequency or a wave packet's energy E dived by $\mathrm{h}$, and $\mathrm{T}$ the oscillation time of the wave. In Ref. [1] it was conjectured that 

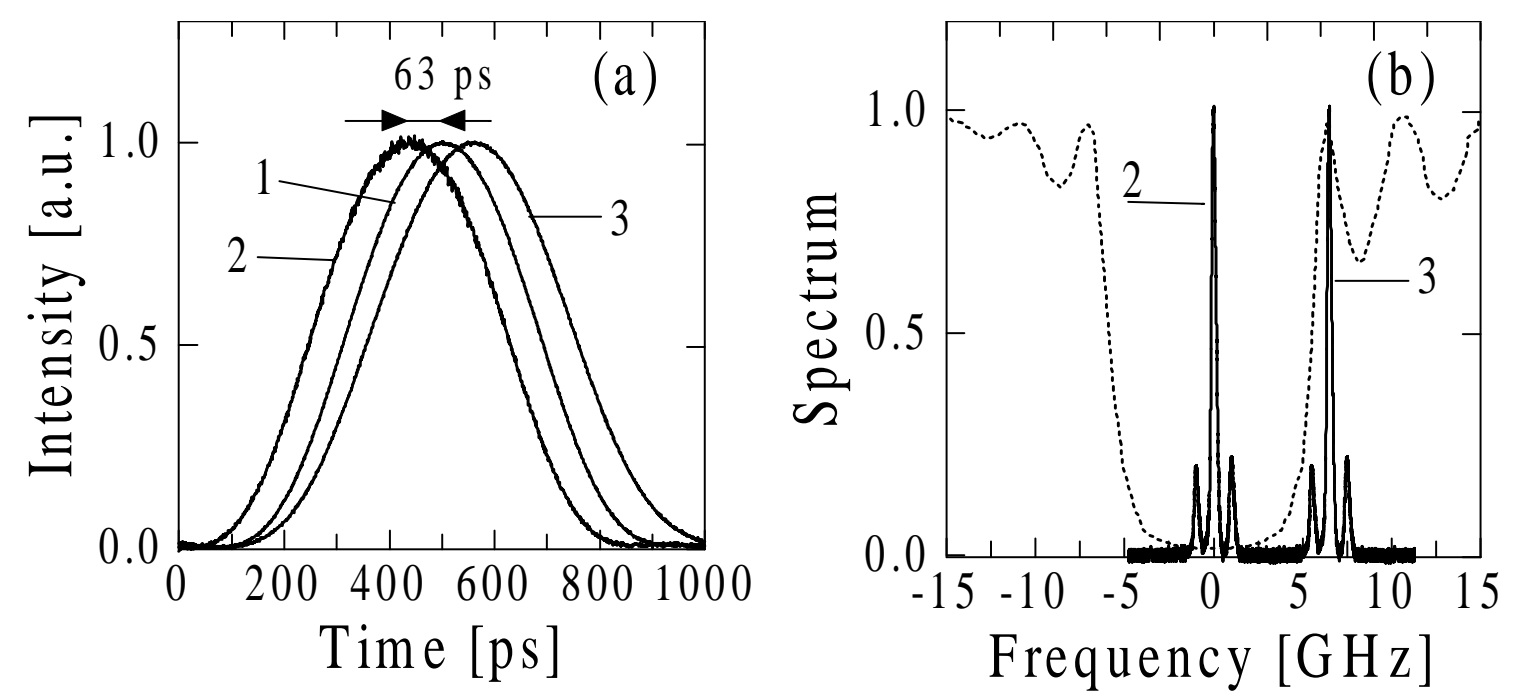

Figure 2: Measured propagation time of three digital signals [7]. (a) Pulse trace 1 was recorded in vacuum. Pulse 2 traversed a photonic lattice in the center of the frequency band gap (see part (b) of the figure), and pulse 3 was recorded for the pulse travelling through the fiber outside the forbidden band gap. The photonic lattice was a periodic dielectric hetero-structure fiber. The IR carrier frequency is $210^{14}$. The signal speed was $2 \mathrm{c}$.

the relation also holds for wave packets with rest mass having in mind the mathematical analogy between the Helmholtz and the Schrödinger equations. Quantum mechanical studies pointed to this conjecture [9, 13, 20].

An argument has been raised recently that a superluminal signal speed can never happen (cf. the comment in Ref. [23]. The superluminal experimental results of digital signals were interpreted analogous to a train loosing at every station a front waggon resulting in a faster train speed of the last arriving waggons. This naive model does not consider that physical signals are frequency band limited as measured and displayed in Fig, 2 and as explained in Ref. 8] for instance. The superluminal signals did not lose any frequency components (that means no loss of waggons as argued in an improper analogy ignoring the QM and QED results [3]). All components of the superluminal signals studied are transmitted and correctly detected as shown in Fig 2. A measurable pulse reshaping did not take place.

All four properties: i) The universal tunneling time. ii) The violation of the Einstein energy relation by the imaginary wave number

$$
E^{2}=(\hbar k c)^{2}+\left(m_{0} c^{2}\right)^{2}
$$

iii) The zero time spreading (non locality), and iv) The non observability of evanescent modes, can be explained by virtual particles in the tunneling process. The Feynman diagram of photonic tunneling is shown in Fig 3 . 
We are used to expect virtual particles in microscopic interaction processes, here we have demonstrated examples in the macroscopic range of a meter in Coulomb and in elastic fields.

\begin{tabular}{|l|l|c|c|}
\hline Table: Tunneling time & & & \\
\hline \hline photonic barriers & reference & $\tau$ & $T=1 / \nu$ \\
\hline \hline \multirow{2}{*}{$\begin{array}{l}\text { frustrated } \\
\text { at doul reflection }\end{array}$} & Haibel/Nimtz & $117 \mathrm{ps}$ & $120 \mathrm{ps}$ \\
\cline { 2 - 4 } & Carey et al. & $\approx 1 \mathrm{ps}$ & $3 \mathrm{ps}$ \\
\cline { 2 - 4 } & Balcou/Dutriaux & $30 \mathrm{fs}$ & $11.3 \mathrm{fs}$ \\
\cline { 2 - 4 } & Mugnai et al. & $134 \mathrm{ps}$ & $100 \mathrm{ps}$ \\
\hline \hline \multirow{2}{*}{ photonic lattice } & Steinberg et al. & $2.13 \mathrm{fs}$ & $2.34 \mathrm{fs}$ \\
\cline { 2 - 4 } & Spielmann et al. & $2.7 \mathrm{fs}$ & $2.7 \mathrm{fs}$ \\
\cline { 2 - 4 } & Nimtz et al. & $81 \mathrm{ps}$ & $115 \mathrm{ps}$ \\
\hline \hline \multirow{2}{*}{$\begin{array}{l}\text { undersized } \\
\text { waveguide }\end{array}$} & Enders/Nimtz & $130 \mathrm{ps}$ & $115 \mathrm{ps}$ \\
\hline \hline \multirow{2}{*}{$\begin{array}{l}\text { electron tunneling (field } \\
\text { emission microscopy, } \\
\text { semiconductor AlGaAs-GaAs) }\end{array}$} & & & \\
\hline \hline \multirow{2}{*}{\begin{tabular}{l} 
acoustic (phonon) tunneling \\
\cline { 2 - 4 }
\end{tabular}} & Sekatskii/Letokhov & $6-8 \mathrm{fs}$ & $>2.43 \mathrm{fs}$ \\
\cline { 2 - 4 } & Yang et al. & $100 \mathrm{fs}$ & $37.5 \mathrm{fs}$ \\
\cline { 2 - 4 } & Robertson et al. & $0.9 \mathrm{~ms}$ & $1.12 \mathrm{~ms}$ \\
\hline
\end{tabular}

\section{References}

[1] Haibel, A. \& Nimtz, G. Universal Relationship of Time and Frequency in Photonic Tunnelling, Ann. Physik 10, 707-712(2001)

[2] Esposito, S., Universal photonic tunneling time, Phys.Rev.E 64,026609-1 $8(2001)$

[3] A A Stahlhofen, A. A. \& Nimtz, G. Evanescent modes are virtual photons. Europhys.Lett.76, 189-195(2006)

[4] Sommerfeld, A., Lectures on Theoretical Physics, Optics. Academic Press Inc. US (1954)

[5] Enders, A. \& Nimtz, G. On superluminal barrier traversal. J. Phys.I, France 2,1693-1698(1992)

[6] Steinberg, A., M., Kwiat, P., G. \& Chiao, R., Y. Measurement of the SinglePhoton Tunneling Time. Phys.Rev.Lett. 71, 708-711(1993)

[7] Longhi, S. Marano, M., Laporta, P. \& Belmonte, M. Superluminal optical pulse propagation in periodic fiber Bragg gratings. Phys.Rev.E 64, 055602-1-4(2001)

[8] Nimtz, G. Do Evanescent Modes Violate Relativistic Causality? The Lecture Notes in Physics, 702, 506-531(2006) 
[9] Hartman, T. Tunneling of a wave packet. J. Appl. Physics 33, 3427-3433(1962)

[10] Enders, A. \& Nimtz,G. Evanescent-mode propagation and quantum tunneling. Phys. Rev. E 48, 632-635(1994)

[11] Carniglia, C., K. \& Mandel, L. Quantization of Evanescent Electromagnetic Waves. Phys.Rev.D 3, 280-296(1971)

[12] Twareque Ali, S. Evanescent Waves in Quantum Electrodynamics with Unquantized Sources. Phys.Rev. D 7, 1668-1675(1973)

[13] Low, F. \& Mende, P., F. A Note on the Tunneling Time Problem. Annals of Physics. 210,380-387(1991)

[14] Wang,Z., Xiong, C. \& He, B. Alternative perspective on photonic tunneling. Phys.Rev.A 75, 013813-1-8(2007)

[15] Nimtz,G. On superluminal tunneling. Progress in Quantum Electronics 27, 417$450(2003)$

[16] Haibel,A., Nimtz,G. \& Stahlhofen,A.A. Frustrated total reflection: The double prisms revisited. Phys. Rev. E 63, 047601 (2001)

[17] Nimtz, G. \& Stahlhofen, A.A., in H. Kleinert and R. Ruffini (eds.): Proceedings of the Eleventh Marcel Grossmann Meeting on General Relativity, World Scientific, Singapore, to appear (2008) and http://arxiv.org/abs/0708.0681

[18] Yang,S. et al.Ultrasound Tunneling through 3D Phononic Crystals, Phys. Rev. Lett. 88, 104301-1-4(2002)

[19] Robertson, W. M. et al. Breaking the sound barrier: Tunneling of acoustic waves through the forbidden transmission region of a one-dimensional acoustic band gap array, Am. J. Phys. 70, 689-693(2002)

[20] Collins, S., Lowe, D. \& Barker, J. J. Phys. C 20, 6213-6232 (1987)

[21] Sekatskii, S. \& Letokhov, V. Electron tunneling time measured by field-emission microscopy. Phys. Rev. B 64, 233311 1-4(2001)

[22] Pereyra,P. Closed formulas for tunneling time in superlattices, Phys.Rev.Lett. 84,1772-1775 (2000)

[23] Steinberg, A. New Scientist, 17 August (2007), NewScientist.com news service 


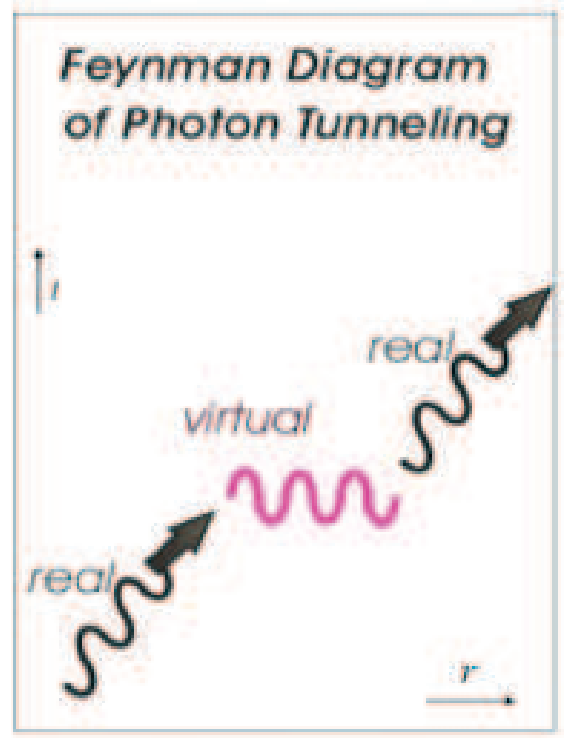

Figure 3: Feynman diagram of evanescent mode or photonic tunneling. Virtual photons connect the observable incoming and transmitted photons. 